\title{
Controle de Urochloa decumbens e Ipomoea triloba pela associação de herbicidas com palha de cana-de-açúcar ${ }^{1}$
}

\author{
Control of Urochloa decumbens and Ipomoea triloba by the association of \\ herbicides with sugarcane straw
}

\author{
Ferdinando Marcos Lima Silva ${ }^{2}$, Sidnei Douglas Cavalieri ${ }^{3}$, Edivaldo Domingues Velini ${ }^{4}$, \\ José Guilherme Ferreira Cordeiro ${ }^{5}$, Alcebíades Rebouças São José ${ }^{6}$
}

\begin{abstract}
Resumo - Objetivou-se com este trabalho avaliar a eficácia de herbicidas associados com palha de cana-de-açúcar no controle de Urochloa decumbens e Ipomoea triloba, após simulação de diferentes períodos de precipitação pluvial. Dois experimentos foram conduzidos em casa de vegetação, em vasos preenchidos com solo, sendo colocados sobre os mesmos o equivalente a 0 , 5 e $10 \mathrm{t} \mathrm{ha}^{-1}$ de palha de cana-de-açúcar, logo após a semeadura das espécies infestantes. Os herbicidas mesotrione, mistura formulada [hexazinone + diuron] e imazapic foram aplicados em pré-emergência nos vasos com ou sem palha e com solo seco. No primeiro experimento, simulouse uma precipitação de $30 \mathrm{~mm}$ a 1 dia após a aplicação (DAA), e no segundo a mesma precipitação aos 14 DAA. A associação da palha de cana-de-açúcar com o herbicida [hexazinone + diuron] não altera sua eficácia. Já a associação com imazapic contribui para o aumento do controle das espécies infestantes. Para o herbicida mesotrione, a simulação de precipitação tardia reduziu o controle de I. triloba.
\end{abstract}

Palavras-chave: cana crua, Saccharum spp., planta daninha

Abstract - This research aimed to evaluate the efficacy of herbicides associated with sugarcane straw on the control of Ipomoea triloba and Urochloa decumbens after simulation of different time periods of rain. Two experiments were conducted in greenhouse in pots filled with soil, being placed on them the equivalent of 0,5 and $10 \mathrm{t} \mathrm{ha}^{-1}$ of sugarcane straw, after weed species sowing. The herbicides mesotrione, formulated mixture of [hexazinone+diuron] and imazapic were applied in pre-emergence on the pots with or without straw and with dry soil. At the first experiment, it was simulated a rainfall of $30 \mathrm{~mm} 1$ day after application (DAA), and at the second

\footnotetext{
${ }^{1}$ Recebido para publicação em 03/10/2011 e aceito em 13/12/2011.

${ }^{2}$ Eng. ${ }^{\circ}$ Agr. ${ }^{\circ}$, M.Sc., Doutorando em Agricultura. Fazenda Experimental Lageado, Núcleo de Pesquisas Avançadas em Matologia (NUPAM), 18603-970, Botucatu-SP. Email: <ferdinando.silva@ yahoo.com.br>;

${ }^{3}$ Eng. $^{\circ}$ Agr. ${ }^{\circ}$, D.Sc., Pesquisador da Embrapa Hortaliças. Rod. BR 060 km 9 (Brasília/Anápolis), 70359-970, Brasília-DF. Email: <cavalieri@ cnph.embrapa.br>;

${ }^{4}$ Eng. $^{\circ}$ Agr. ${ }^{\circ}$, D.Sc. Professor da FCA/UNESP. Núcleo de Pesquisas Avançadas em Matologia (NUPAM), 18603970, Botucatu -SP. Email: <velini@ fca.unesp.br>;

${ }^{5}$ Auxiliar Técnico FCA/UNESP. Núcleo de Pesquisas Avançadas em Matologia (NUPAM), 18603-970 BotucatuSP. Email: <guifca@hotmail.com>;

${ }^{6}$ Eng. ${ }^{\circ}$ Agr. ${ }^{\circ}$, D.Sc., Professor da UESB. Departamento de Fitotecnia e Zootecnia, Estrada do Bem Querer Km 4, 45083-900, Vitória da Conquista-BA. Email: <alreboucas@ gmail.com>
} 
experiment, the same quantity of rain was simulated 14 DAA. The association of sugarcane straw with the herbicide [hexazinone + diuron] does not alter its efficacy. However, the association of sugarcane atraw with imazapic increase the weed control. For the mesotrione herbicide, the late simulation of rainfall reduced I. triloba control.

Key-words: green cane, Saccharum spp., weed

\section{Introdução}

A colheita da cana-de-açúcar (Saccharum spp.) sem a tradicional queima deixa sobre o solo uma espessa camada de palha, que pode superar $20 \mathrm{t} \mathrm{ha}^{-1}$. Tal fato, juntamente com as modificações técnicas necessárias para possibilitar a colheita mecânica da cultura, criou um novo sistema de produção denominado de cana crua (Tofoli et al., 2009). Essa palhada afeta drasticamente e de diferentes maneiras o estabelecimento de plantas daninhas em áreas cultivadas. Dentre elas, citam-se a limitação de variação da temperatura na superfície do solo; a formação de uma barreira física a ser transposta pela planta em crescimento; o aumento da quantidade de microrganismos que podem decompor as sementes dessas plantas; e os possíveis efeitos alelopáticos que inibem a germinação (Negrisoli et al., 2007a).

Apesar disso, algumas plantas daninhas,

como Ipomoea triloba e Euphorbia heterophylla, não têm sua germinação inibida pelas quantidades de palha de cana-de-açúcar que normalmente são encontradas em campo (Martins et al., 1999; Velini e Negrisoli, 2000; Negrisoli et al., 2009). A quantidade de palha produzida pela cultura é função direta das características do clone utilizado, como facilidade de despalha do colmo, hábito de crescimento da touceira, uniformidade em altura e tamanho dos ponteiros, produtividade e desenvolvimento da planta (Manechini, 1997).

As espécies menos afetadas pela presença da palha podem ser selecionadas com o tempo, tornando-se importantes problemas nos canaviais. Portanto, estudos sobre a seleção da flora infestante pela palha são importantes,

pois permitem identificar espécies com potencial de seleção no sistema de colheita de cana crua e estabelecer programas de controle preventivo. Ademais, é de igual importância o desenvolvimento de métodos eficientes para o manejo dessas plantas daninhas pouco sensíveis ao controle pela palha, caso ocorram em elevadas densidades na lavoura (Gravena et al., 2004).

Como as plantas daninhas são um dos pontos críticos no estabelecimento da cultura da cana-de-açúcar, há grande demanda por recursos financeiros para seu controle, sendo a maior parte investida em herbicidas (Quintela et al., 2002). Nessa cultura são usados diversos herbicidas, sendo os pré-emergentes os mais empregados (Medeiros, 2001). Entretanto, um programa integrado de controle dessas espécies ainda é a melhor opção (Gomide, 1993).

A manutenção da palha sobre a superfície do solo pode, simultaneamente, reduzir o potencial de infestação das plantas daninhas e também dificultar o desempenho dos herbicidas, uma vez que a água da precipitação se torna a principal responsável pelo transporte dos herbicidas até a superfície do solo (Maciel \& Velini, 2005; Simoni et al., 2006). Assim, quando um herbicida é aplicado sobre a palha, é interceptado pela superfície dessa e torna-se vulnerável à volatilização e/ou fotólise, até ser lixiviado para o solo (Locke \& Bryson, 1997). Segundo Cavenaghi et al. (2006), com o aumento da quantidade de palha de cana sobre o solo, ocorre diminuição da lixiviação do imazapic pela chuva, principalmente com $20 \mathrm{t} \mathrm{ha}^{-1}$ de palha. $\mathrm{O}$ mesmo resultado foi verificado com amicarbazone (Cavenaghi et al., 2007). 
Diante do exposto, objetivou-se neste trabalho avaliar a eficácia de herbicidas associados com diferentes quantidades de palha de cana-de-açúcar no controle de Urochloa decumbens e I.triloba, após simulação de diferentes períodos de precipitação pluvial posteriores a aplicação dos produtos.

\section{Material e métodos}

Os dois experimentos foram conduzidos em vasos, em casa de vegetação, com temperatura controlada variando de 24 a $28^{\circ} \mathrm{C}$. As unidades experimentais (vasos) possuíam dimensões de 15 × 30 x $12 \mathrm{~cm}$ (largura, comprimento e profundidade), com capacidade para 5 litros e as espécies de plantas daninhas semeadas foram Urochloa decumbens $\mathrm{e}$ I.triloba. A semeadura foi realizada de modo que se obtivesse 25 plantas de cada espécie após a emergência na testemunha.

Como substrato foi usado solo proveniente de um LATOSSOLO VERMELHO Distrófico (LVd) (Embrapa,
2006) que, de acordo com as análises granulométrica e química, apresentava textura média com $20 \%$ de argila, $4 \%$ de silte e $76 \%$ de areia, apresentando na análise química $\mathrm{pH}$ $\mathrm{CaCl}_{2}=4,3 ; \mathrm{H}^{+}+\mathrm{Al}^{3+}, \mathrm{Ca}^{2+}, \mathrm{Mg}^{2+} \mathrm{e} \mathrm{K}^{+}$iguais a 58,$0 ; 10,0 ; 4,0$ e 0,6 mmolc $\mathrm{dm}^{-3}$, respectivamente, $\mathrm{P}=1,0 \mathrm{mg} \mathrm{dm}^{-3}$ e M.O. $=19$ $\mathrm{g} \mathrm{dm}^{-3}$.

Logo após a semeadura das plantas daninhas, foram colocadas sobre os vasos três quantidades de palha de cana-de-açúcar: 0,5 e $10 \mathrm{t} \mathrm{ha} \mathrm{h}^{-1}$. Posteriormente, os herbicidas mesotrione, imazapic e a mistura formulada [hexazinone + diuron] foram aplicados sobre os mesmos, com o solo seco e em pré-emergência, sendo simulada uma precipitação de $30 \mathrm{~mm}$ a 1 DAA e 14 DAA dos herbicidas, onde cada período foi considerado um experimento.

O delineamento experimental foi $\mathrm{o}$ inteiramente casualizado, em esquema fatorial $4 \times 3$ (produtos $x$ quantidade de palha) com quatro repetições, com simulação de precipitação a 1 ou 14 DAA. Os tratamentos encontram-se descritos na Tabela 1.

Tabela 1. Descrição dos tratamentos com herbicidas aplicados em pré-emergência sobre diferentes quantidades de palha $\left(0,5\right.$ e $10 \mathrm{t}_{\text {de }}$ palha $\left.\mathrm{ha}^{-1}\right)$ e simulação de precipitação de $30 \mathrm{~mm}$ a 1 ou 14 dias após a aplicação (DAA). Botucatu, 2009.

\begin{tabular}{cccc}
\hline Tratamento & Herbicida & Dose $\left(\mathrm{g} \mathrm{ha}^{-1}\right)$ & Quantidade de palha $\left(\mathrm{t} \mathrm{ha}^{-1}\right)$ \\
\hline 1 & testemunha & - & 0 \\
2 & mesotrione & 120 & 0 \\
3 & [hexazinone+diuron] & $264+936$ & 0 \\
4 & imazapic & 300 & 0 \\
\hline 5 & testemunha & - & 5 \\
6 & mesotrione & 120 & 5 \\
7 & [hexazinone+diuron] & $264+936$ & 5 \\
8 & imazapic & 300 & 5 \\
9 & testemunha & - & 10 \\
10 & mesotrione & 120 & 10 \\
11 & [hexazinone+diuron] & $264+936$ & 10 \\
12 & imazapic & 300 & 10 \\
\hline
\end{tabular}


A pulverização dos herbicidas e as simulações de precipitação foram realizadas por meio de um equipamento instalado em laboratório, utilizando-se de uma bomba hidráulica de pressão constante e acionamento automático, a qual bombeia água armazenada de um reservatório até a barra e pontas de pulverização responsáveis pela formação de gotas. A barra de simulação de precipitação, situada a $1,45 \mathrm{~m}$ de altura em relação à superfície das unidades experimentais, é constituída por três pontas de pulverização, modelo TK-SS-20 de alta vazão, espaçadas de $0,5 \mathrm{~m}$ e posicionadas de forma a propiciar uniformidade de aplicação.

Esse sistema foi operado com velocidade de deslocamento de $0,187 \mathrm{~km} \mathrm{~h}^{-1} \mathrm{e}$ pressão de trabalho de $0,81 \mathrm{kgf} \mathrm{s}^{-2}$, sendo cada lâmina de água aplicada correspondente a 2,5 $\mathrm{mm}$. Essas especificações proporcionaram a produção de gotas artificiais de precipitação com diâmetro mediano volumétrico (DMV) de 1.140 micrometros.

A barra de pulverização de herbicidas era constituída por quatro pontas de pulverização, modelo XR 110.02 VS, espaçadas de $0,5 \mathrm{~m}$ entre si e posicionadas a $0,5 \mathrm{~m}$ de altura em relação à superfície dos vasos. Para as pulverizações dos herbicidas, o sistema foi operado com velocidade de deslocamento de $3,6 \mathrm{~km} \mathrm{~h}^{-1}$, o que correspondeu a 45 Hertz no modulador de frequência, com volume de aplicação correspondente a $200 \mathrm{~L} \mathrm{ha}^{-1}$. O equipamento foi operado com pressão constante de 1,5 bar pressurizado por ar comprimido.

As avaliações de controle das espécies de plantas daninhas foram realizadas por meio de uma escala visual e percentual de notas, em que 0 (zero) corresponde a nenhuma injúria e 100 (cem) à morte das plantas, segundo escala da ALAM (1974), sendo realizadas aos 14, 21 e 28 dias após a precipitação (DAP). Ao final dos experimentos (28 DAP) as plantas foram coletadas para a determinação da matéria seca (MS).

Em ambos os experimentos, os dados foram submetidos à análise de variância e as médias comparadas pelo teste " $\mathrm{t}$ " $(\mathrm{p}<0,05)$, utilizando-se o programa estatístico SISVAR (Ferreira, 1999).

\section{Resultados e discussão}

O controle de $U$. decumbens com ocorrência de precipitação a 1 dia após a aplicação - DAA (Tabela 2) obtido pela aplicação da mistura formulada [hexazinone + diuron] foi significativamente superior aos demais herbicidas ao final do experimento (28 dias após precipitação - DAP) e também nas primeiras avaliações (14 e 21 DAP), exceto para o controle com $5 \mathrm{t}$ de palha ha ${ }^{-1}$ aos 14 DAP, que não apresentou diferença para o imazapic.

O herbicida imazapic apresentou adequado controle dessa espécie daninha, com notas ao final do experimento em torno de $90 \%$ de controle. Hernandez et al. (2001) também observaram menor controle de $U$. decumbens pelo imazapic, apesar do herbicida ser registrado para a mesma, mas a sua ação pode ter sido atrapalhada pelo excesso de umidade do solo, já que esse foi mantido úmido após a aplicação dos herbicidas e parte do imazapic pode ter sido lixiviado.

O mesotrione não controlou $U$. decumbens, porém foi ajudado pela presença da palha, apresentando notas de controle, quando na presença de palha, significativamente superiores ao tratamento sem palha. A eficácia dos demais herbicidas não foi afetada pela ausência ou presença de palha nas quantidades estudas (Tabela 2). 
Tabela 2. Porcentagem de controle (\%) de U. decumbens por herbicidas aos 14, 21 e 28 dias após simulação de precipitação de $30 \mathrm{~mm}$ a 1 dia após a aplicação (DAA) com 0,5 e $10 \mathrm{t}$ de palha ha ${ }^{-}$ 1 . Botucatu, 2009.

\begin{tabular}{|c|c|c|c|c|c|c|c|c|c|}
\hline \multirow{3}{*}{ Tratamento } & \multicolumn{3}{|c|}{14 DAP } & \multicolumn{3}{|c|}{$21 \mathrm{DAP}$} & \multicolumn{3}{|c|}{28 DAP } \\
\hline & \multicolumn{3}{|c|}{ palha $\left(\mathrm{t} \mathrm{ha}^{-1}\right)$} & \multicolumn{3}{|c|}{ palha $\left(\mathrm{t} \mathrm{ha}^{-1}\right)$} & \multicolumn{3}{|c|}{ de palha $\left(\mathrm{t} \mathrm{ha}^{-1}\right)$} \\
\hline & 0 & 5 & 10 & 0 & 5 & 10 & 0 & 5 & 10 \\
\hline testemunha & $0,00 \mathrm{dA}$ & $0,00 \mathrm{cA}$ & $0,00 \mathrm{cA}$ & $0,00 \mathrm{dA}$ & $0,00 \mathrm{dA}$ & $0,00 \mathrm{dA}$ & $0,00 \mathrm{dA}$ & $0,00 \mathrm{dA}$ & $0,00 \mathrm{dA}$ \\
\hline mesotrione & $56,25 \mathrm{cB}$ & $75,00 \mathrm{bA}$ & $78,75 \mathrm{bA}$ & $43,75 \mathrm{cB}$ & $66,25 \mathrm{cA}$ & $66,25 \mathrm{cA}$ & $37,50 \mathrm{cB}$ & $82,50 \mathrm{cA}$ & $76,25 \mathrm{cA}$ \\
\hline imazapic & $72,50 \mathrm{bB}$ & $82,50 \mathrm{aA}$ & 83,75 bA & $86,25 \mathrm{bA}$ & $87,75 \mathrm{bA}$ & 88,25 bA & $89,00 \mathrm{bA}$ & $90,50 \mathrm{bA}$ & $90,50 \mathrm{bA}$ \\
\hline [hex+diuron] & $92,75 \mathrm{aA}$ & $85,70 \mathrm{aB}$ & $95,00 \mathrm{aA}$ & $96,25 \mathrm{aA}$ & $95,25 \mathrm{aA}$ & $99,00 \mathrm{aA}$ & $98,50 \mathrm{aA}$ & $98,50 \mathrm{aA}$ & $99,25 \mathrm{aA}$ \\
\hline $\mathrm{F}(\mathrm{T})$ & & $1152,17 *$ & & & $1154,36^{*}$ & & & $1130,96^{*}$ & \\
\hline $\mathrm{F}(\mathrm{P})$ & & $18,73^{*}$ & & & $10,87 *$ & & & $30,52 *$ & \\
\hline$F(T) x(P)$ & & $9,45^{*}$ & & & $8,08 *$ & & & $27,35^{*}$ & \\
\hline C.V. $(\%)$ & & 6,96 & & & 7,32 & & & 7,24 & \\
\hline D.M.S.(T)x(P) & & 6,01 & & & 6,38 & & & 6,6 & \\
\hline
\end{tabular}

Na Tabela 3, constata-se que os herbicidas mesotrione e [hexazinone + diuron] apresentaram excelente controle de I. triloba para todos os níveis de palha com precipitação a 1 DAA. Para o herbicida imazapic, a presença de palha incrementou significativamente o controle dessa espécie, mas foi inferior aos outros dois herbicidas. A formulação [hexazinone + diuron] apresentou excelente controle de I. triloba na condição sem palha já aos 14 DAP, e o mesmo não ocorreu para os herbicidas mesotrione $\mathrm{e}$ imazapic. Em trabalho anterior, Cavenaghi et al. (2006) observou que o aumento da quantidade de palha de cana sobre o solo promove a diminuição da lixiviação do imazapic pela chuva, principalmente nas maiores quantidades de palha, como $20 \mathrm{t} \mathrm{ha}^{-1}$.

Tabela 3. Porcentagem de controle (\%) de I. triloba por herbicidas aos 14, 21 e 28 dias após simulação de precipitação de $30 \mathrm{~mm}$ a 1 dia após a aplicação (DAA) com 0,5 e $10 \mathrm{t}$ de palha ha ${ }^{-}$ 1 . Botucatu, 2009.

\begin{tabular}{|c|c|c|c|c|c|c|c|c|c|}
\hline \multirow{3}{*}{ Tratamento } & \multicolumn{3}{|c|}{14 DAP } & \multicolumn{3}{|c|}{$21 \mathrm{DAP}$} & \multicolumn{3}{|c|}{28 DAP } \\
\hline & \multicolumn{3}{|c|}{ palha $\left(\mathrm{t} \mathrm{ha}^{-1}\right)$} & \multicolumn{3}{|c|}{ palha $\left(\mathrm{t} \mathrm{ha}^{-1}\right)$} & \multicolumn{3}{|c|}{ palha $\left(\mathrm{t} \mathrm{ha}^{-1}\right)$} \\
\hline & 0 & 5 & 10 & 0 & 5 & 10 & 0 & 5 & 10 \\
\hline testemunha & $0,00 \mathrm{dA}$ & $0,00 \mathrm{cA}$ & $0,00 \mathrm{cA}$ & $0,00 \mathrm{cA}$ & $0,00 \mathrm{cA}$ & $0,00 \mathrm{cA}$ & $0,00 \mathrm{cA}$ & $0,00 \mathrm{cA}$ & $0,00 \mathrm{cA}$ \\
\hline mesotrione & $84,00 \mathrm{bB}$ & $93,00 \mathrm{aA}$ & $96,00 \mathrm{aA}$ & $96,00 \mathrm{aA}$ & $98,00 \mathrm{aA}$ & $96,25 \mathrm{abA}$ & $99,50 \mathrm{aA}$ & $100 \mathrm{aA}$ & $98,75 \mathrm{abA}$ \\
\hline imazapic & $35,00 \mathrm{cC}$ & $73,75 \mathrm{bB}$ & $86,25 \mathrm{bA}$ & $45,00 \mathrm{bC}$ & $88,75 \mathrm{bB}$ & $93,00 \mathrm{bA}$ & $61,25 \mathrm{bB}$ & $97,00 \mathrm{bA}$ & $97,50 \mathrm{bA}$ \\
\hline [hex+diuron] & $96,00 \mathrm{aA}$ & $96,50 \mathrm{aA}$ & $99,00 \mathrm{aA}$ & $96,00 \mathrm{aB}$ & $99,00 \mathrm{aA}$ & $99,00 \mathrm{aA}$ & $99,25 \mathrm{aA}$ & $100 \mathrm{aA}$ & $100 \mathrm{aA}$ \\
\hline $\mathrm{F}(\mathrm{T})$ & & $993,66^{*}$ & & & $3923,29 *$ & & & 10736,53 & \\
\hline $\mathrm{F}(\mathrm{P})$ & & $49,21 *$ & & & $134,79 *$ & & & $174,55^{*}$ & \\
\hline $\mathrm{F}(\mathrm{T}) \mathrm{x}(\mathrm{P})$ & & $25,85^{*}$ & & & $101,22 *$ & & & $166,94 *$ & \\
\hline C.V. (\%) & & 7,72 & & & 3,78 & & & 2,25 & \\
\hline D.M.S.(T)x(P) & & 7,00 & & & 3,66 & & & 2,30 & \\
\hline
\end{tabular}

Médias seguidas de mesma letra minúscula na coluna e maiúscula na linha, não diferem significativamente entre si pelo teste "t" ( $\mathrm{p}<0,05)$. Doses $\left(\mathrm{g} \mathrm{ha}^{-1}\right)$ : mesotrione (120), [hexazinone+diuron] $(264+936)$ e imazapic (300).

O eficiente controle de plantas daninhas do gênero Ipomoea tem grande importância no atual manejo da cana-de-açúcar, pois algumas espécies desse gênero tendem são de difícil controle nas áreas de cana-de-açúcar conduzidas no sistema de colheita mecanizada. Azânia et al. (2002) afirmaram que, com até 15 t ha ${ }^{-1}$ de palha, a emergência de I. triloba, $I$. 
hederifolia e $I$. nil não foi alterada. Tem sido constatada redução nas populações dessas plantas daninhas somente na presença de $20 \mathrm{t}$ $\mathrm{ha}^{-1}$. Quanto às espécies I. quamoclit e Merremia cissoides, o efeito negativo da palha de cana-de-açúcar foi observado nas quantidades de 15 e $20 \mathrm{t} \mathrm{ha}^{-1}$, mas não inferior a $10 \mathrm{t} \mathrm{ha}^{-1}$.

Neste estudo, a palha não reduziu a eficácia da mistura [hexazinone + diuron] no controle de I. triloba, no entanto, em estudo realizado por Monquero et al. (2009c) com a mesma planta daninha, os pesquisadores observaram que quantidades crescentes de palha reduziram o controle de I. triloba por essa mistura, fato esse explicado pela baixa precipitação no ensaio após a aplicação dos herbicidas $(9 \mathrm{~mm})$, mostrando a importância da precipitação para lavar o produto da palha e levá-lo até o solo. Em outro estudo de eficácia de herbicidas aplicados em pré-emergência sobre a palha de cana-de-açúcar, Monquero et al. (2009b) relataram que o herbicida mesotrione teve a eficácia alterada pela presença de palha, sendo positiva no controle de Bidens pilosa e negativa no controle de $I$. quamoclit.

Negrisoli et al. (2007b) avaliando o herbicida tebuthiuron, também observaram que a aplicação sobre a palha de cana-de-açúcar resultou em excelente controle da espécie $I$. triloba, com médias de $100 \%$ nesse mesmo período, quando ocorreu precipitação de 20 $\mathrm{mm}$ após 24 horas da pulverização. Esse resultado também foi observado por Negrisoli et al. (2009) com o herbicida oxyfluorfen e por Toledo et al. (2009) com o herbicida amicarbazone.

No que diz respeito à matéria seca (MS) das plantas daninhas (Tabela 4), não houve diferença significativa entre os tratamentos avaliados, exceto para o controle de $U$. decumbens sem palha, apesar de haver diferença de MS entre os tratamentos e essa diferença acompanhar as notas de controle. As diferentes quantidades de palha somente influenciaram na MS da testemunha, reduzindo-a no caso da $U$. decumbens e $I$. triloba.

Tabela 4. Matéria seca (g) de $U$. decumbens e I. triloba aos 28 dias após a aplicação (DAA) de herbicidas e simulação de precipitação de $30 \mathrm{~mm}$ a 1 DAA com 0,5 e $10 \mathrm{t}$ de palha ha ${ }^{-1}$. Botucatu, 2009

\begin{tabular}{|c|c|c|c|c|c|c|}
\hline \multirow{3}{*}{ Tratamento } & \multicolumn{3}{|c|}{ Urochloa decumbens } & \multicolumn{3}{|c|}{ Ipomoea triloba } \\
\hline & \multicolumn{3}{|c|}{ palha $\left(\mathrm{t} \mathrm{ha}^{-1}\right)$} & \multicolumn{3}{|c|}{ palha $\left(\mathrm{t} \mathrm{ha}^{-1}\right)$} \\
\hline & 0 & 5 & 10 & 0 & 5 & 10 \\
\hline testemunha & $4,3552 \mathrm{cB}$ & $3,2573 \mathrm{Ba}$ & $2,9430 \mathrm{bA}$ & $0,1288 \mathrm{aB}$ & $0,1733 \mathrm{bB}$ & $0,6065 \mathrm{bA}$ \\
\hline mesotrione & $1,2300 \mathrm{bA}$ & $0,4685 \mathrm{aA}$ & $0,5003 \mathrm{aA}$ & $0,0000 \mathrm{aA}$ & $0,0000 \mathrm{aA}$ & $0,0020 \mathrm{aA}$ \\
\hline imazapic & $0,2440 \mathrm{aA}$ & $0,1738 \mathrm{aA}$ & $0,1838 \mathrm{aA}$ & $0,0855 \mathrm{aA}$ & $0,0090 \mathrm{aA}$ & $0,0013 \mathrm{aA}$ \\
\hline [hex+diuron] & $0,0025 \mathrm{aA}$ & $0,0055 \mathrm{aA}$ & $0,0013 \mathrm{aA}$ & $0,0050 \mathrm{aA}$ & $0,0000 \mathrm{aA}$ & $0,0000 \mathrm{aA}$ \\
\hline $\mathrm{F}(\mathrm{T})$ & \multicolumn{3}{|c|}{$91,67^{*}$} & \multicolumn{3}{|c|}{$31,68^{*}$} \\
\hline $\mathrm{F}(\mathrm{P})$ & \multicolumn{3}{|c|}{$4,13 *$} & \multicolumn{3}{|c|}{$6,91 *$} \\
\hline$F(T) x(P)$ & \multicolumn{3}{|c|}{1,44} & \multicolumn{3}{|c|}{$9,49 *$} \\
\hline C.V. $(\%)$ & \multicolumn{3}{|c|}{53,03} & \multicolumn{3}{|c|}{106,94} \\
\hline $\operatorname{DMS}(\mathrm{T}) \mathrm{x}(\mathrm{P})$ & \multicolumn{3}{|c|}{0,8471} & \multicolumn{3}{|c|}{0,1292} \\
\hline
\end{tabular}

Médias seguidas de mesma letra minúscula na coluna e maiúscula na linha, não diferem significativamente entre si pelo teste " $\mathrm{t}$ " $(\mathrm{p}<0,05)$. Doses $\left(\mathrm{g} \mathrm{ha}^{-1}\right)$ : mesotrione (120), [hexazinone+diuron] (264+936) e imazapic (300) 
Por ocasião da ocorrência de precipitação tardia afetou a ação dos precipitação aos 14 DAA, os tratamentos apresentaram comportamento semelhante ao experimento com precipitação a 1 DAA, herbicidas. Como a redução de $10 \%$ no controle de $U$. decumbens pelo herbicida imazapic no tratamento sem palha (Tabela 5). exceto para alguns casos específicos, em que a

Tabela 5. Porcentagem de controle (\%) de $U$. decumbens por herbicidas aos 14, 21 e 28 dias após simulação de precipitação de $30 \mathrm{~mm}$ a 14 dias após a aplicação (DAA) com 0,5 e $10 \mathrm{t}$ de palha ha $^{-1}$. Botucatu, 2009.

\begin{tabular}{|c|c|c|c|c|c|c|c|c|c|}
\hline \multirow{3}{*}{ Tratamento } & \multicolumn{3}{|c|}{14 DAP } & \multicolumn{3}{|c|}{$21 \mathrm{DAP}$} & \multicolumn{3}{|c|}{28 DAP } \\
\hline & \multicolumn{3}{|c|}{ palha $\left(\mathrm{t} \mathrm{ha}^{-1}\right)$} & \multicolumn{3}{|c|}{ palha $\left(\mathrm{t} \mathrm{ha}^{-1}\right)$} & \multicolumn{3}{|c|}{ palha $\left(\mathrm{t} \mathrm{ha}^{-1}\right)$} \\
\hline & 0 & 5 & 10 & 0 & 5 & 10 & 0 & 5 & 10 \\
\hline testemunha & $0,00 \mathrm{dA}$ & $0,00 \mathrm{dA}$ & $0,00 \mathrm{cA}$ & $0,00 \mathrm{dA}$ & $0,00 \mathrm{dA}$ & $0,00 \mathrm{dA}$ & $0,00 \mathrm{dA}$ & $0,00 \mathrm{dA}$ & $0,00 \mathrm{dA}$ \\
\hline mesotrione & $16,25 \mathrm{cB}$ & $65,00 \mathrm{cA}$ & $65,00 \mathrm{bA}$ & $23,75 \mathrm{cC}$ & $76,25 \mathrm{cA}$ & $50,00 \mathrm{cB}$ & $35,00 \mathrm{cC}$ & $73,75 \mathrm{cA}$ & $45,00 \mathrm{cB}$ \\
\hline imazapic & $58,75 \mathrm{bB}$ & $85,75 \mathrm{bA}$ & $89,50 \mathrm{aA}$ & $66,25 \mathrm{bB}$ & $84,50 \mathrm{bA}$ & $87,75 \mathrm{bA}$ & $80,00 \mathrm{bB}$ & $85,75 \mathrm{bAB}$ & $90,50 \mathrm{bA}$ \\
\hline [hex+diuron] & $85,75 \mathrm{aB}$ & $93,50 \mathrm{aA}$ & $94,75 \mathrm{aA}$ & $94,25 \mathrm{aA}$ & $97,00 \mathrm{aA}$ & $97,50 \mathrm{aA}$ & $98,00 \mathrm{aA}$ & $100 \mathrm{aA}$ & $98,25 \mathrm{aA}$ \\
\hline $\mathrm{F}(\mathrm{T})$ & & $807,73^{*}$ & & & $1135,33^{*}$ & & & $1361,92 *$ & \\
\hline $\mathrm{F}(\mathrm{P})$ & & $101,60 *$ & & & $75,33 *$ & & & $31,75^{*}$ & \\
\hline $\mathrm{F}(\mathrm{T}) \mathrm{x}(\mathrm{P})$ & & $26,28 *$ & & & $33,42 *$ & & & $23,24 *$ & \\
\hline C.V. $(\%)$ & & 9,05 & & & 7,69 & & & 7,02 & \\
\hline D.M.S.(T) $x(P)$ & & 7,07 & & & 6,22 & & & 5,93 & \\
\hline
\end{tabular}

A redução no controle de I. triloba, quantidade de palha apresentou incremento entre 3,8 e 4,3\%, nas quantidades de 5 e $10 \mathrm{t}$ crescente na eficácia do herbicida para $\mathrm{o}$ de palha ha ${ }^{-1}$ pelo herbicida mesotrione (Tabela controle de U. decumbens (Tabela 5).

6). No caso do imazapic, o aumento da

Tabela 6. Porcentagem de controle (\%) de I. triloba por herbicidas aos 14, 21 e 28 dias após simulação de precipitação de $30 \mathrm{~mm}$ a 14 dias após a aplicação (DAA) com 0,5 e $10 \mathrm{t}$ de palha ha ${ }^{-1}$. Botucatu, 2009.

\begin{tabular}{|c|c|c|c|c|c|c|c|c|c|}
\hline \multirow{3}{*}{ Tratamento } & \multicolumn{3}{|c|}{14 DAP } & \multicolumn{3}{|c|}{$21 \mathrm{DAP}$} & \multicolumn{3}{|c|}{28 DAP } \\
\hline & \multicolumn{3}{|c|}{ palha $\left(\mathrm{t} \mathrm{ha}^{-1}\right)$} & \multicolumn{3}{|c|}{ palha $\left(\mathrm{t} \mathrm{ha}^{-1}\right)$} & \multicolumn{3}{|c|}{ palha $\left(\mathrm{t} \mathrm{ha}^{-1}\right)$} \\
\hline & 0 & 5 & 10 & 0 & 5 & 10 & 0 & 5 & 10 \\
\hline testemunha & $0,00 \mathrm{dA}$ & $0,00 \mathrm{cA}$ & $0,00 \mathrm{cA}$ & $0,00 \mathrm{dA}$ & $0,00 \mathrm{cA}$ & $0,00 \mathrm{bA}$ & $0,00 \mathrm{cA}$ & $0,00 \mathrm{bA}$ & $0,00 \mathrm{cA}$ \\
\hline mesotrione & $61,25 \mathrm{bB}$ & $92,25 \mathrm{aA}$ & $88,75 \mathrm{bA}$ & $85,75 \mathrm{bB}$ & $96,50 \mathrm{abA}$ & $95,25 \mathrm{aA}$ & $100 \mathrm{aA}$ & $95,75 \mathrm{aAB}$ & $95,00 \mathrm{bB}$ \\
\hline imazapic & $32,50 \mathrm{cC}$ & $71,25 \mathrm{bB}$ & $88,25 \mathrm{bA}$ & $57,50 \mathrm{cB}$ & $92,00 \mathrm{bA}$ & $97,00 \mathrm{aA}$ & $66,25 \mathrm{bB}$ & $97,00 \mathrm{aA}$ & $97,50 \mathrm{abA}$ \\
\hline [hex+diuron] & $95,75 \mathrm{aA}$ & $99,00 \mathrm{aA}$ & $99,00 \mathrm{aA}$ & $95,50 \mathrm{aA}$ & $99,00 \mathrm{aA}$ & $99,00 \mathrm{aA}$ & $99,50 \mathrm{aA}$ & $100 \mathrm{aA}$ & $100 \mathrm{aA}$ \\
\hline $\mathrm{F}(\mathrm{T})$ & & $688,00 *$ & & & $1389,38^{*}$ & & & $2707,66^{*}$ & \\
\hline $\mathrm{F}(\mathrm{P})$ & & $67,94 *$ & & & $47,18^{*}$ & & & $23,98^{*}$ & \\
\hline $\mathrm{F}(\mathrm{T}) \mathrm{x}(\mathrm{P})$ & & $23,71 *$ & & & $21,03 *$ & & & $35,53^{*}$ & \\
\hline C.V. $(\%)$ & & 9,31 & & & 6,26 & & & 4,47 & \\
\hline D.M.S.(T)x $(\mathrm{P})$ & & 8,10 & & & 6,11 & & & 4,54 & \\
\hline
\end{tabular}


Independente da simulação de precipitação a 1 ou 14 DAA, o herbicida mesotrione não controlou a $U$. decumbens, apresentando notas significativamente inferiores aos demais produtos testados (Tabelas 2 e 5). Tal fato já era esperado, pois o mesotrione não é recomendado para o controle dessa espécie (Rodrigues \& Almeida, 2005).

Os dados de controle de I. triloba (Tabela 6) pelo herbicida mesotrione foram semelhantes aos encontrados por Monquero et al. (2009a), com tendência de redução do controle quando passa-se da situação sem palha para a condição com palha de cana-deaçúcar.

Nos dados de matéria seca das plantas daninhas (Tabela 7) não houve diferença estatística entre os herbicidas aplicados, exceto para o controle de $U$. decumbens, nas condições sem palha e com a maior quantidade de palha em que o herbicida mesotrione foi inferior aos tratamentos com imazapic e [hexazinone + diuron].

De forma geral, os resultados encontrados corroboram com os obtidos por Costa (2001) que relatou que os herbicidas residuais, para exercerem sua atividade sobre as plantas infestantes, precisam ser introduzidos diretamente ao solo, o que é proporcionado pelo carreamento por meio da água de precipitação, em função da solubilidade do herbicida, quando da aplicação sobre a palhada de cana-de-açúcar. E demonstram a necessidade de mais estudos com outras moléculas herbicidas e espécies invasoras.

Tabela 7. Matéria seca (g) de U. decumbens e I. triloba aos 28 dias após a aplicação (DAA) de herbicidas e simulação de precipitação de $30 \mathrm{~mm}$ a 14 DAA com 0 , 5 e $10 \mathrm{t}$ de palha ha ${ }^{-1}$. Botucatu, 2009

\begin{tabular}{|c|c|c|c|c|c|c|}
\hline \multirow[t]{2}{*}{ Tratamento } & \multicolumn{3}{|c|}{$\begin{array}{c}\text { Urochloa decumbens } \\
\text { palha }\left(\mathrm{t} \mathrm{ha}^{-1}\right)\end{array}$} & \multicolumn{3}{|c|}{$\begin{array}{c}\text { Ipomoea triloba } \\
\text { palha }\left(\mathrm{t} \mathrm{ha}^{-1}\right)\end{array}$} \\
\hline & 0 & 5 & 10 & 0 & 5 & 10 \\
\hline testemunha & $4,3553 \mathrm{cB}$ & $3,2573 \mathrm{bA}$ & $2,9430 \mathrm{bA}$ & $0,1988 \mathrm{bB}$ & $0,1733 \mathrm{bB}$ & $0,6065 \mathrm{bA}$ \\
\hline mesotrione & $1,8155 \mathrm{bB}$ & $0,7043 \mathrm{aA}$ & $2,1820 \mathrm{bB}$ & $0,0175 \mathrm{aA}$ & $0,0035 \mathrm{aA}$ & $0,0040 \mathrm{aA}$ \\
\hline imazapic & $0,3552 \mathrm{aA}$ & $0,2228 \mathrm{aA}$ & $0,1528 \mathrm{aA}$ & $0,0690 \mathrm{aA}$ & $0,0050 \mathrm{aA}$ & $0,0045 \mathrm{aA}$ \\
\hline [hex+diuron] & $0,0055 \mathrm{aA}$ & $0,0018 \mathrm{aA}$ & $0,0045 \mathrm{aA}$ & $0,0000 \mathrm{aA}$ & $0,0000 \mathrm{aA}$ & $0,0000 \mathrm{aA}$ \\
\hline $\mathrm{F}(\mathrm{T})$ & \multicolumn{3}{|c|}{$65,97^{*}$} & \multicolumn{3}{|c|}{$31,22 *$} \\
\hline $\mathrm{F}(\mathrm{P})$ & \multicolumn{3}{|c|}{2,92} & \multicolumn{3}{|c|}{$7,08^{*}$} \\
\hline $\mathrm{F}(\mathrm{T}) \mathrm{x}(\mathrm{P})$ & \multicolumn{3}{|c|}{1,08} & \multicolumn{3}{|c|}{$9,20^{*}$} \\
\hline C.V. $(\%)$ & \multicolumn{3}{|c|}{51,53} & \multicolumn{3}{|c|}{107,39} \\
\hline $\operatorname{DMS}(\mathrm{T}) \mathrm{x}(\mathrm{P})$ & \multicolumn{3}{|c|}{0,9852} & \multicolumn{3}{|c|}{0,1299} \\
\hline
\end{tabular}

Médias seguidas de mesma letra minúscula na coluna e maiúscula na linha, não diferem significativamente entre si pelo teste " $\mathrm{t}$ " ( $\mathrm{p}<0,05)$. Doses $\left(\mathrm{g} \mathrm{ha}^{-1}\right)$ : mesotrione (120), [hexazinone+diuron] $(264+936)$ e imaza pic (300). 


\section{Conclusões}

A associação da palha de cana-deaçúcar com o herbicida [hexazinone + diuron] não altera sua eficácia. Por outro lado, com o herbicida imazapic ocorre o aumento do controle das espécies infestantes. Para o herbicida mesotrione, a simulação de precipitação tardia reduziu o controle de $I$. triloba.

\section{Agradecimentos}

Os autores agradecem ao Conselho Nacional de Pesquisa (CNPq) pela concessão de bolsa de mestrado ao primeiro autor.

\section{Referências}

ASSOCIATION LATINOAMERICANA DE MALEZAS - ALAM. Recomendaciones sobre unificación de los sistemas de evaluación em ensayos de control de malezas. ALAM, v.1, p.35-38, 1974.

AZÂNIA, A.A.P.M. et al. Interferência da palha de cana-de-açúcar (Saccharum spp.) na emergência de espécies de plantas daninhas da família convolvulaceae. Planta Daninha, v.20, n.2, p.207-212, 2002.

CAVENAGHI, A. L. et al. Dinâmica do herbicida amicarbazone aplicado sobre a palha de cana-de-açúcar. Planta Daninha, v.25, n.4, p.831-837, 2007.

CAVENAGHI, A. L. et al. Dinâmica do herbicida imazapic aplicado sobre a palha de cana-de-açúcar. In: CONGRESSO BRASILEIRO DA CIÊNCIA DAS PLANTAS DANINHAS, 25., 2006a. Resumos... Brasília: SBCPD/ UNB/ Embrapa Cerrados, 2006. p. 360.

COSTA, E.A.D. Efeito de adjuvantes na dinâmica de ametryn em palha de cana-deaçúcar. 2001, 81f. Tese (Doutorado em Agronomia/Proteção de Plantas) - Faculdade de Ciências Agronômicas, Universidade Estadual Paulista "Júlio de Mesquita Filho", Botucatu.

EMBRAPA. 2006. Sistema Brasileiro de Classificação dos Solos. 2 ed. Rio de Janeiro: EMBRAPA, $412 \mathrm{p}$.

FERREIRA, D.F. Sistema de análise de variância (Sisvar). versão 4.6. Lavras: Departamento de Ciências Exatas, 1999. (CDROM).

HERNANDEZ， D.D.; ALVES， P.L.C.A.; MARTINS, J.V.F. Influência do resíduo de colheita de cana-de-açúcar sem queima sobre a eficiência dos herbicidas imazapic e imazapic+ pendimenthalin. Planta Daninha, v.19, n.3, p.419-426, 2001.

GOMIDE, M.B. Potencialidades alelopáticas dos restos culturais de dois cultivares de cana-de-açúcar (Saccharum spp.), no controle de algumas plantas daninhas. 1993. 96f. Tese (Doutoramento em Fitotecnia) Escola Superior de Agricultura Luiz de Queiroz, Piracicaba.

GRAVENA, R. et al. Controle de plantas daninhas através da palha de cana-de-açúcar associada à mistura dos herbicidas trifloxysulfuron sodium + ametrina. Planta Daninha, v. 22, n.3, p.419-427, 2004.

LOCKE, M.A.; BRYSON, C.T. Herbicide-soil interaction in reduced tillage and plant residue management systems. Weed Science, v.45, p.307-320, 1997.

MACIEL, C.D.G.; VELINI, E. D. Simulação do caminhamento da água da precipitação e herbicidas em palhadas utilizadas em sistemas de plantio direto. Planta Daninha, v.23, n.3, p.471-481, 2005.

MANECHINI, C. Manejo da cana crua. In: SEMINÁRIO COPERSUCAR DE TECNOLOGIA AGRONÔMICA, 7., 1997, Piracicaba. Anais... Piracicaba: 1997. p. 30927. 
MARTINS, D. et al. Emergência em campo de dicotiledôneas infestantes em solo coberto com palha de cana-de-açúcar. Planta Daninha, v.17, n.1, p.151-161, 1999.

MEDEIROS, D. Efeitos da palha de cana-deaçúcar (Saccharum spp.) sobre o manejo de plantas daninhas e dinâmica do banco de sementes. 2001. 126f. Dissertação (Mestrado em Agronomia) - Universidade de São Paulo, Piracicaba.

MONQUERO, P.A. et al. Eficácia de herbicidas aplicados em diferentes épocas e espécies daninhas em área de cana-de-açúcar colhida mecanicamente. Planta Daninha, v.27, n.2, p.309-317, 2009a.

MONQUERO, P.A. et al. Eficácia de herbicidas aplicados em diferentes épocas sobre B. pilosa e I. quamoclit em área de canade-açúcar colhida mecanicamente. Planta Daninha, v.27, n.3, p.563-570, 2009 b.

MONQUERO, P.A. et al. Eficácia de herbicidas em diferentes quantidades de palha de cana-de-açúcar no controle de Ipomoea grandifolia. Bragantia, v.68, n.2, p.367-372, 2009c.

NEGRISOLI, E. et al. Controle de plantas daninhas pelo amicarbazone aplicado na presença de palha de cana-de-açúcar. Planta Daninha, v.25, n.3, p. 603-611, 2007a.

NEGRISOLI, E. et al. Associação do herbicida tebuthiuron com a cobertura de palha no controle de plantas daninhas no sistema de cana-crua. Planta Daninha, v.25, n.3, p.621$628,2007 \mathrm{~b}$.

NEGRISOLI, E. et al. Eficácia do herbicida oxyfluorfen com a cobertura de palha no controle de plantas daninhas. Planta Daninha, v.27, n.1, p.197-203, 2009.

QUINTELA, A.C.R. et al. Controle de plantas daninhas em cana crua (cultivar RB835089) no sistema integrado palhiço, herbicida e vinhaça. STAB, v.20, n.4, p.38-42, 2002.
RODRIGUES, B.N.; ALMEIDA, F.S. Guia de herbicidas. 5a edição. Londrina: Grarfmake, 2005. 592p.

SIMONI, F. et al. Eficácia de imazapic e sulfentrazone sobre Cyperus rotundus em diferentes condições de precipitação e palha de cana-de-açúcar. Planta Daninha, v.24, n.4, p.769-778, 2006.

TOFOLI, G.R. et al. Dinâmica do tebuthiuron em palha de cana-de-açúcar. Planta Daninha, v.27, n.4, p.815-821, 2009.

TOLEDO, R.E.B. et al. Eficácia do herbicida amicarbazone aplicado sobre a palha ou no solo no controle de plantas daninhas na cultura da cana-de-açúcar. Planta Daninha, v.27, n.2, p.319-326, 2009.

VELINI, E.D.; NEGRISOLI, E. Controle de plantas daninhas em cana crua. In: CONGRESSO BRASILEIRO DA CIÊNCIA DAS PLANTAS DANINHAS, 22., 2000, Foz de Iguaçu. Palestras... Foz de Iguaçu: Sociedade Brasileira da Ciência das Plantas Daninhas, 2000. p. 148-164. 